\title{
A Spherical Particle Settling Towards a Corrugated Wall
}

\author{
K. Lamzoud, R. Assoudi, F. Bouisfi, M. Chaoui
}

Based on the assumption of low Reynolds number, the flow around a spherical particle settling towards a corrugated wall in a fluid at rest is described by Stokes equations. In the case of the small amplitude of wall roughness, the asymptotic expansion coupled with the Lorentz reciprocal theorem are used to derive analytical expressions of the hydrodynamic effects due to wall roughness. The evolution of these effects in terms of roughness parameters and also the sphere-wall distance are discussed.

Keywords: Stokes equations, low Reynolds number, roughness effects, asymptotic expansion, Lorentz reciprocal theorem

\section{Introduction}

The problems of the motion of spherical particles suspended in a viscous fluid limited by a wall have various applications, such as the separation technics like field-flow fraction (FFF) [1] and capillary hydrodynamic chromatography (CHDC) [2], small scale transport phenomena, microfluidics, and biological flow fields [3, 4]. It should be noted that much work has been devoted to the study of spherical particles moving near smooth walls, see, for example, [5]. In this context, the problems of flows around spherical particles moving in translation and rotation, in the vicinity of a smooth wall with slip or non-slip conditions, have been solved in different

Received April 30, 2019

Accepted June 14, 2019

The work was supported by Faculty of science, Department of physics, Moulay Ismail University.

Khalid Lamzoud

lamzoud.moeva@gmail.com

Redouane Assoudi

r.assoudi@gmail.com

Firdouss Bouisfi

firdaous.bouisfi@gmail.com

Mohamed Chaoui

chaouimohammad@gmail.com

Faculty of sciences, Moulay Ismail University,

Marjane 2, BP:298, Meknes 50050, Morocco

RUSSIAN JOURNAL OF NONLINEAR DYNAMICS, 2019, 15(2), 125-134 
articles. Thus, with the non-slip condition, the problem of translation and rotation parallel to a smooth wall of a smooth spherical particle has been treated by O'Neill $[6,7]$ by using the bispherical coordinates method. It was reviewed by Goldman [8], who also provided lubrication formulas for the case where the gap between the sphere and the wall is small compared to the sphere's radius. This problem was later reviewed by Chaoui and Feuillebois [9], who developed a precise technique for calculating the force and torque coefficients in bispherical coordinates. With the slip condition $[10,11]$ on the wall, this problem of the motion of spherical particles, in a viscous fluid bounded by a smooth wall, has been reviewed by several authors [12-14]. The majority of the theoretical studies model the surface of the wall as a smooth surface, whereas a surface, whatever its manufacturing process, is not a perfectly smooth surface. All of these surface defects constitute the wall roughness. This roughness certainly has an influence either at the macroscopic scale or at the microscopic scale [15]. Few results are available for hydrodynamic interactions between particles and rough walls, although they are ubiquitous and of practical importance. Falade and Brenner [16] carried out a theoretical study of the motion of a translational and rotational spherical particle, in a fluid at rest, near a weakly deformable wall by using an asymptotic expansion based on a dimensionless coefficient $\beta=a / R \ll 1$, where $a$ is the sphere's radius and $R$ is the radius associated with the curvature of the nearest point of the wall from the sphere surface. Smart and Leighton $[17,18]$ measured the hydrodynamic effect of the roughness of a sphere surface moving perpendicular to a smooth wall. Some of their spheres have been roughened by sticking very small spheres on their surfaces. Lecoq et al [19] as well as Lecoq [20] considered the alternative problem of a sphere moving perpendicularly to a corrugated wall. They have prepared the walls with a predefined roughness, while the roughness of the sphere was neglected. They considered a periodic roughness (which varies in one direction) under the assumption that the period is weak compared to the radius of the sphere, and their study is limited to the lubrication region. In a previous investigation [21], we have studied the problem of a freely spherical particle, in a shear flow parallel to a rough wall. The roughness considered is periodic and varies in one direction. We have calculated the effects generated by the flow of the second order due to the wall by using the asymptotic expansion coupled with the Lorentz reciprocal theorem. In this study, we will keep the same roughness profile, but we will consider the problem of a spherical particle settling towards a rough wall in a fluid at rest. We will look for analytical expressions as well as numerical values of the effects generated by the wall roughness.

\section{Description of the problem}

Consider a rigid spherical particle, of radius $a$, moving with a velocity $\tilde{\mathbf{U}}_{p}$ towards a corrugated wall in a fluid at rest (Fig. 1).

The roughness of the wall considered here is periodic and changes in one direction. The wall profile is described in the right-handed system of rectangular Cartesian coordinates $(\widetilde{X}, \widetilde{Y}, \widetilde{Z})$ by

$$
\widetilde{Z}_{p}=a \varepsilon R(\widetilde{X}),
$$

where $\varepsilon$ is a dimensionless amplitude of roughness, and $R(\widetilde{X})$ is a normalized function which describes the profile roughness, its expansion in Fourier series being expressed as

$$
\mathcal{R}(\widetilde{X})=c_{0}+\sum_{n=1}^{p}\left(c_{n} \cos (n \omega \widetilde{X})+s_{n} \sin (n \omega \widetilde{X})\right),
$$



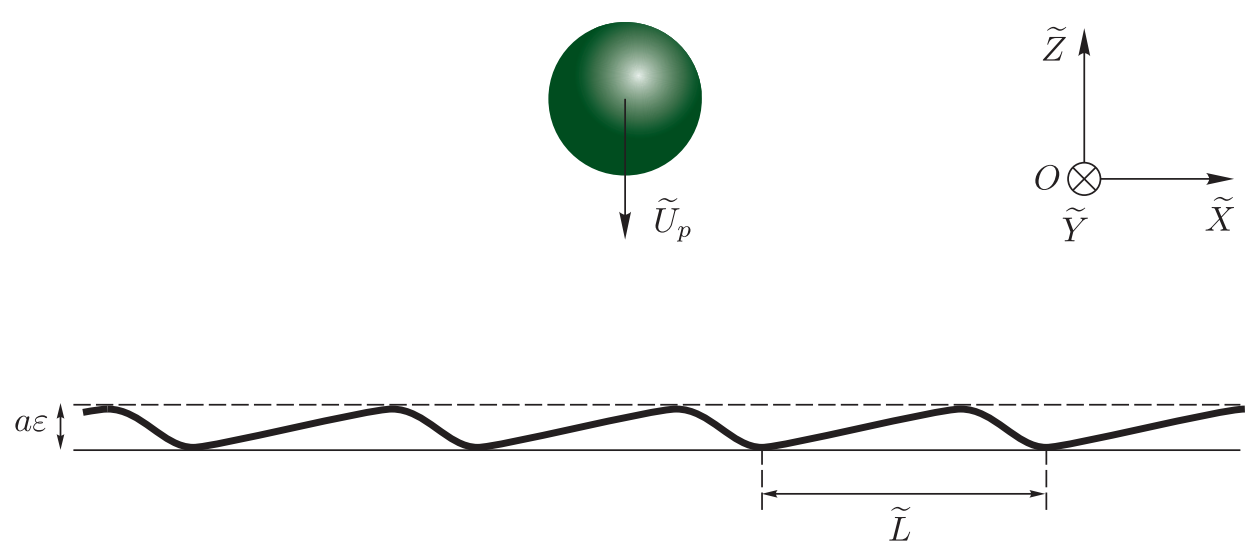

Fig. 1. A spherical particle settling with a velocity $\tilde{\mathbf{U}}_{p}$ towards a rough wall.

where $\omega=2 \pi / \widetilde{\mathrm{L}}$ is a positive constant and

$$
\begin{gathered}
c_{0}=\frac{1}{\widetilde{\mathrm{L}}} \int_{0}^{\widetilde{\mathrm{L}}} R(\widetilde{X}) d \widetilde{X} \\
c_{n}=\frac{2}{\widetilde{\mathrm{L}}} \int_{0}^{\widetilde{\mathrm{L}}} R(\widetilde{X}) \cos (n \omega \widetilde{X}) d \widetilde{X}, \quad s_{n}=\frac{2}{\widetilde{\mathrm{L}}} \int_{0}^{\widetilde{\mathrm{L}}} R(\widetilde{X}) \sin (n \omega \widetilde{X}) d \widetilde{X} .
\end{gathered}
$$

Taking $p=3$ in the Fourier expansion (1.2) is sufficient to describe the profile as shown in Fig. 1. Let $\left(\tilde{\mathbf{v}}_{S t}, \tilde{p}_{S t}\right)$ be the velocity and pressure fields of the flow around the sphere. By assumption the low Reynolds number $R_{e}=\frac{\rho_{f} a V_{c}}{\mu_{f}} \ll 1, \rho_{f}$ and $\mu_{f}$ are, respectively, the fluid density and dynamic viscosity and $V_{c}$ is a reference velocity, and the flow around the sphere is described by Stokes equations

$$
\left\{\begin{array} { c } 
{ \mu _ { f } \tilde { \nabla } ^ { 2 } \widetilde { \mathbf { v } } _ { S t } = \tilde { \nabla } \widetilde { p } _ { S t } } \\
{ \tilde { \nabla } \cdot \widetilde { \mathbf { v } } _ { S t } = 0 }
\end{array} \text { with } \left\{\begin{array}{ll}
\widetilde{\mathbf{v}}_{S t}=\tilde{\mathbf{U}}_{p} & \text { on the sphere surface, } \\
\widetilde{\mathbf{v}}_{S t}=\mathbf{0} & \text { on the rough wall, } \\
\widetilde{\mathbf{v}}_{S t}=\mathbf{0} & \text { at infinity from the particle. }
\end{array}\right.\right.
$$

\section{Low roughness amplitude}

By considering the case where the amplitude of the roughness is very small compared to the sphere's radius, $\varepsilon \ll 1$, the velocity and pressure fields of the flow around the sphere can be developed as follows:

$$
\begin{aligned}
& \widetilde{\mathbf{v}}_{S t}=\widetilde{\mathbf{v}}_{S t}^{(0)}+\varepsilon \widetilde{\mathbf{v}}_{S t}^{(1)}+O\left(\varepsilon^{2}\right) \\
& \widetilde{p}_{S t}=\widetilde{p}_{S t}^{(0)}+\varepsilon \widetilde{p}_{S t}^{(1)}+O\left(\varepsilon^{2}\right)
\end{aligned}
$$

with

- $\left(\widetilde{\mathbf{v}}_{S t}^{(0)}, \widetilde{p}_{S t}^{(0)}\right)$ : the velocity and the pressure fields generated by the settling spherical particle towards a smooth wall located at $\tilde{Z}=0$.

- $\left(\widetilde{\mathbf{v}}_{S t}^{(1)}, \widetilde{p}_{S t}^{(1)}\right)$ : the velocity and the pressure fields generated by the wall roughness. 
The same for the sphere's velocity

where

$$
\tilde{\mathbf{U}}_{p}=\tilde{\mathbf{U}}_{p}^{(0)}+\varepsilon \tilde{\mathbf{U}}_{p}^{(1)}+O\left(\varepsilon^{2}\right),
$$

- $\tilde{\mathbf{U}}_{p}^{(0)}$ is the settling velocity of the sphere in the absence of roughness, it has just one component $\tilde{\mathbf{U}}_{p}^{(0)}=-\tilde{U}_{p}^{(0)} \mathbf{i}_{z}$.

- $\tilde{\mathbf{U}}_{p}^{(1)}$ is the correction due to the roughness of the wall, it can be described by $\tilde{\mathbf{U}}_{p}^{(1)}=$ $=\tilde{U}_{x}^{(1)} \mathbf{i}_{x}+\tilde{U}_{z}^{(1)} \mathbf{i}_{z}$.

The analytical expressions of the first-order flow, $\left(\widetilde{\mathbf{v}}_{S t}^{(0)}, \widetilde{p}_{S t}^{(0)}\right)$, have already been calculated in $[22]$ by using the bispherical coordinates method.

\subsection{Flow of second order $\left(\widetilde{\mathbf{v}}_{S t}^{(1)}, \widetilde{\boldsymbol{p}}_{S t}^{(1)}\right)$}

By substituting the asymptotic expansions (2.1) and (2.2) into Eq. (1.3). And by linearity of the model, the flow of the second order due to the roughness is described also by the Stokes equations

$$
\left\{\begin{aligned}
\mu_{f} & \tilde{\nabla}^{2} \widetilde{\mathbf{v}}_{S t}^{(1)} \\
& =\tilde{\nabla} \widetilde{p}_{S t}^{(1)} \\
\tilde{\nabla} \cdot \widetilde{\mathbf{v}}_{S t}^{(1)} & =0
\end{aligned}\right.
$$

associated with the boundary conditions

$$
\begin{cases}\widetilde{\mathbf{v}}_{S t}^{(1)}=\tilde{\mathbf{U}}_{p}^{(1)} & \text { on the sphere surface, } \\ \widetilde{\mathbf{v}}_{S t}^{(1)}=\mathbf{0} & \text { at infinity from the sphere's center, } \\ \widetilde{\mathbf{v}}_{S t}^{(1)}=-a \mathcal{R}(\widetilde{x}) \frac{\partial \widetilde{\mathbf{v}}_{S t}^{(0)}}{\partial \widetilde{z}} & \text { on a fictious plane wall } \tilde{Z}=0\end{cases}
$$

The third condition, on the fictious smooth wall, is obtained by a Taylor development of the velocity field $\widetilde{\mathbf{v}}_{S t}$ of perturbed flow near a fictious plane wall located under the roughness at $\tilde{Z}=0$. This condition expresses, on the smooth wall, the velocity field of the second-order flow in terms of the velocity field of the first-order flow. This technique is called the flattening of the boundary condition, it allows us to convert the no-slip condition on a surface with relief to a fictitious smooth wall with a slip condition that appears variable. This technique has already been used in several previous studies $[21,23]$. The complexity of these boundary conditions makes the analytical resolution of velocity and pressure fields difficult. The Lorentz reciprocity theorem is a shortcut that allows us to find the second-order effects due to the flow generated by the roughness without needing to solve it analytically.

\subsection{Lorentz reciprocal theorem and the second-order effects}

In the same way, we can define the force and the torque exerted on the spherical particle during its movement by

$$
\begin{aligned}
& \widetilde{\mathbf{F}}_{S t}=\widetilde{\mathbf{F}}_{S t}^{(0)}+\varepsilon \widetilde{\mathbf{F}}_{S t}^{(1)}+O\left(\varepsilon^{2}\right), \\
& \widetilde{\mathbf{C}}_{S t}=\widetilde{\mathbf{C}}_{S t}^{(0)}+\varepsilon \widetilde{\mathbf{C}}_{S t}^{(1)}+O\left(\varepsilon^{2}\right)
\end{aligned}
$$


with

- $\left(\widetilde{\mathbf{F}}_{S t}^{(0)}, \widetilde{\mathbf{C}}_{S t}^{(0)}\right)$ the force and torque exerted on the sphere in the case of a smooth wall.

- $\left(\widetilde{\mathbf{F}}_{S t}^{(1)}, \widetilde{\mathbf{C}}_{S t}^{(1)}\right)$ the force and torque due to the second-order flow generated by the wall roughness.

The flow of the first order $\left(\widetilde{\mathbf{v}}_{S t}^{(0)}, \widetilde{p}_{S t}^{(0)}\right)$ generated by a spherical particle settling towards a smooth wall is axisymmetric, so its influence on the particle is expressed only by a drag force $\widetilde{\mathbf{F}}_{S t}^{(0)}$ and no torque $\widetilde{\mathbf{C}}_{S t}^{(0)}$. The analytical expression and numerical values of this force are calculated in previous studies $[22,23]$. The symmetry of the system with respect to the plane $\tilde{X}=0$ leads to cancelling the component following $\tilde{Y}$ of the force due to the flow of second order

$$
\widetilde{\mathbf{F}}_{S t}^{(1)}=\widetilde{F}_{x, S t}^{(1)} \mathbf{i}_{x}+\widetilde{F}_{z, S t}^{(1)} \mathbf{i}_{z} .
$$

For the same reasons, the $\tilde{X}$ and $\tilde{Z}$ components of the torque vanish so that:

$$
\widetilde{\mathbf{C}}_{S t}^{(1)}=\widetilde{C}_{y, S t}^{(1)} \mathbf{i}_{y} .
$$

Determination of the analytical expression of these three effects is through the use of the Lorentz reciprocity theorem. The reciprocal flows used verify the following conditions on the plane wall and at infinity:

$$
\left.\tilde{\mathbf{u}}^{*}\right|_{\tilde{Z}=0}=\mathbf{0},\left.\quad \tilde{\mathbf{u}}^{*}\right|_{\mathcal{S}_{\infty}}=\mathbf{0} .
$$

On the sphere surface

- $\left.\tilde{\mathbf{u}}^{*}\right|_{\mathcal{S}}=\tilde{U}_{x}^{*} \mathbf{i}_{x}$ in the case of a reciprocal flow generated by a translational particle near a smooth wall in fluid at rest.

- $\left.\tilde{\mathbf{u}}^{*}\right|_{\mathcal{S}}=-\tilde{U}_{z}^{*} \mathbf{i}_{z}$ in the case of a reciprocal flow generated by a settling spherical particle towards a smooth wall in fluid at rest.

- $\left.\tilde{\mathbf{u}}^{*}\right|_{\mathcal{S}}=\tilde{\Omega}_{y}^{*} \wedge \tilde{\mathbf{r}}$ in the case of the flow generated by a rotational particle in a fluid at rest near a smooth wall.

Let $\left(\tilde{\mathbf{u}}^{*}, \sigma^{*}\right)$ be the velocity field and stress tensor of the reciprocal flow, and $\left(\tilde{\mathbf{v}}_{S t}^{(1)}, \sigma_{S t}^{(1)}\right)$ the velocity field and the stress tensor of the physical flow. The Lorentz reciprocal theorem is written as

$$
\int_{\partial D} \tilde{\mathbf{v}}_{S t}^{(1)} \cdot \tilde{\boldsymbol{\sigma}}^{*} \cdot \tilde{\mathbf{d S}}=\int_{\partial D} \tilde{\mathbf{u}}^{*} \cdot \tilde{\boldsymbol{\sigma}}_{S t}^{(1)} \cdot \tilde{\mathbf{d} \mathbf{S}}
$$

with $\partial D=\mathcal{P}+\mathcal{S}+\mathcal{S}_{\infty}$, where $\mathcal{P}$ is the virtual plane wall surface, $\mathcal{S}$ is the sphere surface and $\mathcal{S}_{\infty}$ is a surface at infinity. By using the boundary conditions, on the sphere surface and on the fictitious plane wall surface as well as at infinity, for the physical and reciprocal flows, we end up with

$$
\left\{\begin{array}{c}
\tilde{U}_{x}^{*} \widetilde{F}_{x, S t}^{(1)}=-a \widetilde{L}_{S t}^{*, t}+\tilde{U}_{x}^{(1)} \widetilde{F}_{x}^{*, t} \\
-\tilde{U}_{z}^{*} \widetilde{F}_{z, S t}^{(1)}=-a \widetilde{L}_{S t}^{*, s t}+\tilde{U}_{z}^{(1)} \widetilde{F}_{x}^{*, s t} \\
\tilde{\Omega}_{y}^{*} \widetilde{C}_{y, S t}^{(1)}=-a \widetilde{L}_{S t}^{*, r}+\tilde{U}_{x}^{(1)} \widetilde{F}_{x}^{*, r}
\end{array}\right.
$$


where

$$
\begin{aligned}
\widetilde{L}_{S t}^{*, t} & =\int_{\mathcal{P}} \mathcal{R}(\widetilde{x})\left[\frac{\partial \tilde{\mathbf{v}}_{S t}^{(0)}}{\partial \tilde{z}}\right]_{\tilde{z}=0} \cdot\left[\tilde{\boldsymbol{\sigma}}^{*, t} \cdot \mathbf{i}_{z}\right] \tilde{d S} \\
\widetilde{L}_{S t}^{*, r} & =\int_{\mathcal{P}} \mathcal{R}(\widetilde{x})\left[\frac{\partial \tilde{\mathbf{v}}_{S t}^{(0)}}{\partial \tilde{z}}\right]_{\tilde{z}=0} \cdot\left[\tilde{\boldsymbol{\sigma}}^{*, r} \cdot \mathbf{i}_{z}\right] \tilde{d S} \\
\widetilde{L}_{S t}^{*, S t} & =\int_{\mathcal{P}} \mathcal{R}(\widetilde{x})\left[\frac{\partial \tilde{\mathbf{v}}_{S t}^{(0)}}{\partial \tilde{z}}\right]_{\tilde{z}=0} \cdot\left[\tilde{\boldsymbol{\sigma}}^{*, s t} \cdot \mathbf{i}_{z}\right] \tilde{d S},
\end{aligned}
$$

$\widetilde{F}_{x}^{*, t}, \widetilde{F}_{z}^{*, s t}$ and $\widetilde{F}_{x}^{*, r}$ are the drag forces generated by reciprocal flows due to, respectively, translational, settling and rotational particle in the fluid at rest near a smooth wall. Their analytical expressions are given in terms of dimensionless coefficients of the flows of the first order in [9, 22].

We have a system consisting of three equations with five unknowns, which makes direct resolution impossible. To remedy this problem, we will impose either the force and torque to extract the expression of the speed of the sphere or the speed to extract the expressions of the force and couple.

$1^{\text {st }}$ case: the sphere velocity is imposed, so that it is of order $1 . \tilde{\mathbf{U}}_{p}=\tilde{\mathbf{U}}_{p}^{(0)}=-\tilde{U}_{z}^{(0)} \mathbf{i}_{z}$, from (2.10), the force and torque are expressed by

$$
\begin{aligned}
\widetilde{F}_{x, S t}^{(1)} & =-\frac{a}{\tilde{U}_{x}^{*}} \widetilde{L}_{S t}^{*, t} \\
\widetilde{F}_{z, S t}^{(1)} & =\frac{a}{\widetilde{U}_{z}^{*}} \widetilde{L}_{S t}^{*, s t} \\
\widetilde{C}_{y, S t}^{(1)} & =-\frac{a}{\tilde{\Omega}_{y}^{*}} \widetilde{L}_{S t}^{*, r}
\end{aligned}
$$

$2^{\text {nd }}$ case: the force and torque are imposed to be of the first order $\left(\widetilde{\mathbf{F}}_{S t}, \widetilde{\mathbf{C}}_{S t}\right)=\left(\widetilde{\mathbf{F}}_{S t}^{(0)}, \mathbf{0}\right)$, the velocity of the second order of the sphere is expressed as

$$
\begin{aligned}
\widetilde{U}_{x, S t}^{(1)} & =\frac{a}{\tilde{F}_{x}^{*}, t} \widetilde{L}_{S t}^{*, t} \\
\widetilde{U}_{z, S t}^{(1)} & =\frac{a}{\tilde{F}_{z}^{*, t}} \widetilde{L}_{S t}^{*, s t} .
\end{aligned}
$$

The terms $\widetilde{L}_{S t}^{*, t}, \widetilde{L}_{S t}^{*, s t}$ and $\widetilde{L}_{S t}^{*, r}$ are made dimensionless by using the quantities $\left(\mu_{f} V_{c}^{*} \tilde{U}_{z}\right)$, with $V_{c}^{*}$ the characteristic velocity of the reciprocal flow which is used to calculate the desired effect. For the drag force $\widetilde{F}_{z}^{*, s t}$, the lift forces $\widetilde{F}_{x}^{*, t}$ and $\widetilde{F}_{x}^{*, r}$ are made dimensionless by using, respectively, the quantities $6 \pi a \mu_{f} \tilde{U}_{z},-6 \pi a \mu_{f} \tilde{U}_{x}^{*}$ and $6 \pi a^{2} \mu_{f} \tilde{\Omega}_{y}^{*}$.

All dimensionless quantities will be denoted without tilde.

\subsection{Dimensionless expressions of the effects generated by the wall roughness}

By using the quantities above, the dimensionless expressions of the second-order effects are written as follows:

- Force and torque

$$
\begin{aligned}
f_{x, S t}^{(1)} & =-\frac{1}{6 \pi} L_{S t}^{*, t}, \\
f_{z, S t}^{(1)} & =\frac{1}{6 \pi} L_{S t}^{*, s t},
\end{aligned}
$$




$$
c_{y, S t}^{(1)}=\frac{1}{8 \pi} L_{S t}^{*, r}
$$

- Velocity

$$
\begin{aligned}
U_{x, S t}^{(1)} & =-\frac{1}{6 \pi f_{x, T}^{(0)}} L_{S t}^{*, t}, \\
U_{z, S t}^{(1)} & =\frac{1}{6 \pi f_{z, S t}^{(0)}} L_{S t}^{*, s t},
\end{aligned}
$$

where $f_{x, S t}^{(1)}, f_{z, S t}^{(1)}$ and $c_{y, S t}^{(1)}$ are, respectively, the lift, drag and torque dimensionless coefficients due to the roughness. $U_{x, S t}^{(1)}$ and $U_{z, S t}^{(1)}$ are the dimensionless horizontal and vertical components of the sphere velocity generated by the wall roughness. $f_{x, T}^{(0)}$ is the dimensionless drag coefficient exerted on the translational spherical particle in a fluid at rest parallel to the smooth wall [9], and $f_{z, S t}^{(0)}$ is the dimensionless drag coefficient exerted on the spherical particle settling towards a smooth wall [22].

\section{Results and discussion}

In this section, we will evaluate the numerical values of the second-order effects generated by the roughness in terms of $l / a$, the distance between the sphere center and the fictitious plane wall located below the roughness, the period $\mathrm{L}$ as well as the relative position $\mathrm{T} / \mathrm{L}$ of the sphere along a period. The calculation of the terms $L_{S t}^{*, t}, L_{S t}^{*, s t}$ and $L_{S t}^{*, r}$ was done using the Gauss - Laguerre quadrature method.

Numerical results (Fig. 2a) show that the roughness generates a drag force with a very large coefficient $f_{z, S t}^{(1)}$ and a lift $f_{x, S t}^{(1)}$ which is low in front of the drag, the two coefficients reaching their maximum, respectively, $f_{z, S t}^{(1)}=2.75$ and $f_{x, S t}^{(1)}=0.5$, when the particle is in the lubrication zone. The roughness generates also a torque $c_{z, S t}^{(1)}$ which has a very low intensity. As expected, these effects begin to diminish rapidly as the distance between the particle and the wall increases.

Figure $2 \mathrm{~b}$ represents the evaluation of the second-order kinematic torsor in terms of the distance between the particle center and the fictitious wall. The numerical results show that the roughness introduces corrections on the kinematic torsor of the sphere. It generates a velocity of z-direction of the dimensionless coefficient $U_{z, S t}^{(1)}$ and a migration velocity $U_{x, S t}^{(1)}$, both of them are very important when the particle approaches the wall. The positive sign of $U_{z, S t}^{(1)}$ means that the roughness leads to weakening the sedimentation movement of the sphere.

These variations can be interpreted physically: when the particle approaches the rough wall, the fluid becomes trapped in the cavities below the particle, which leads to an increase in pressure in this area; this explains the increase of these effects in the case of small gaps.

Figures $3 \mathrm{a}$ and $3 \mathrm{~b}$ represent the evaluation of second-order effects in terms of the roughness period $\mathrm{L}$. These variations can be interpreted physically by the change of the distance separating the sphere surface and the peaks of the closet slopes of roughness when the period changes.

Figures $4 \mathrm{a}$ and $4 \mathrm{~b}$ represent the evaluation of the second-order effects as a function of the relative position $\mathrm{T} / \mathrm{L}$ of the sphere along one period. As expected, these effects vary in a periodic way. The coefficients $f_{x, S t}^{(1)}$ and $U_{x, S t}^{(1)}$ vanish at the positions $\mathrm{T} / \mathrm{L}=0.1$ and $\mathrm{T} / \mathrm{L}=0.65$, which means that the sphere-rough-wall system becomes symmetric and the wall in these positions behaves like a fictitious smooth one. 


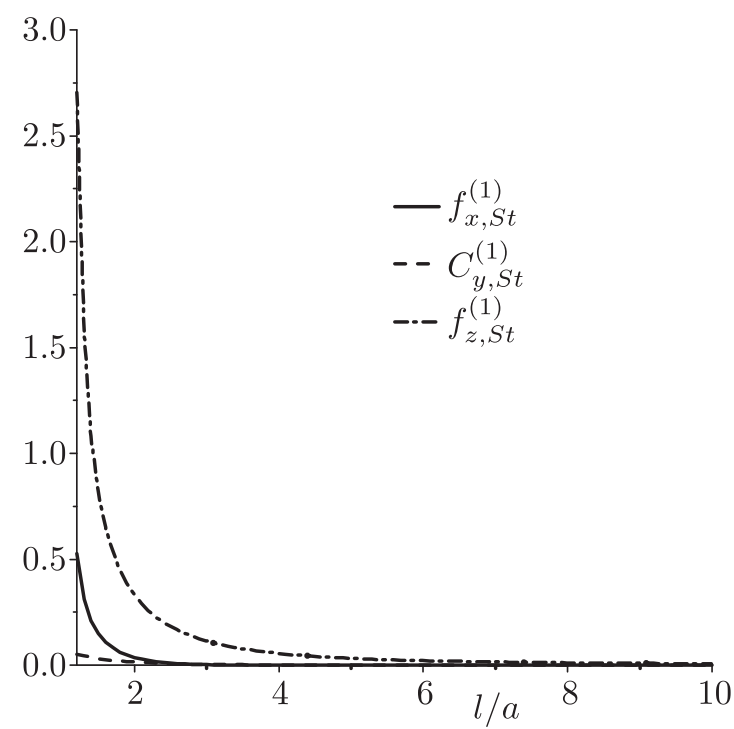

(a)

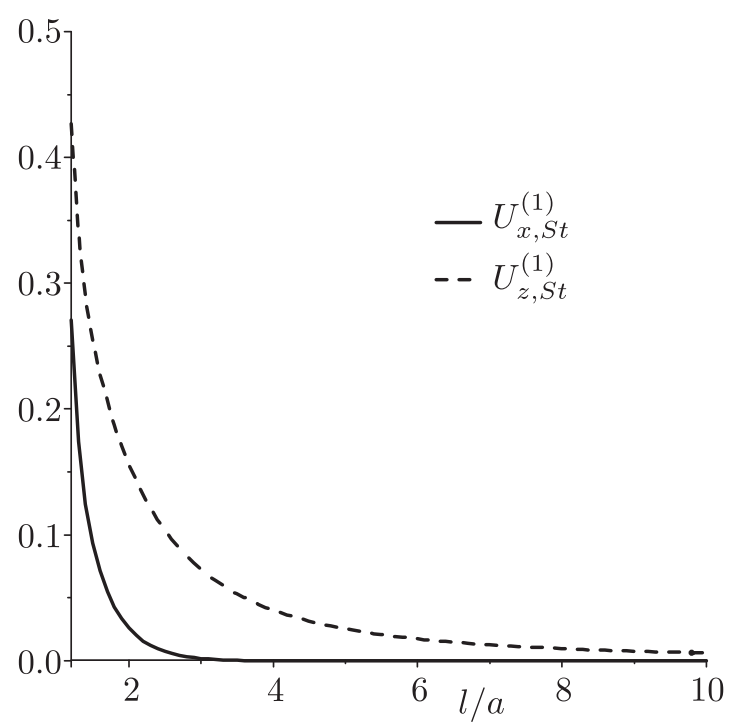

(b)

Fig. 2. (a) Lift, torque and drag coefficients and (b) Horizontal and vertical components of velocity; in terms of $l / a$. At $\mathrm{T}=0$ with $\mathrm{L}=4$ and $\delta=3 / 4$.

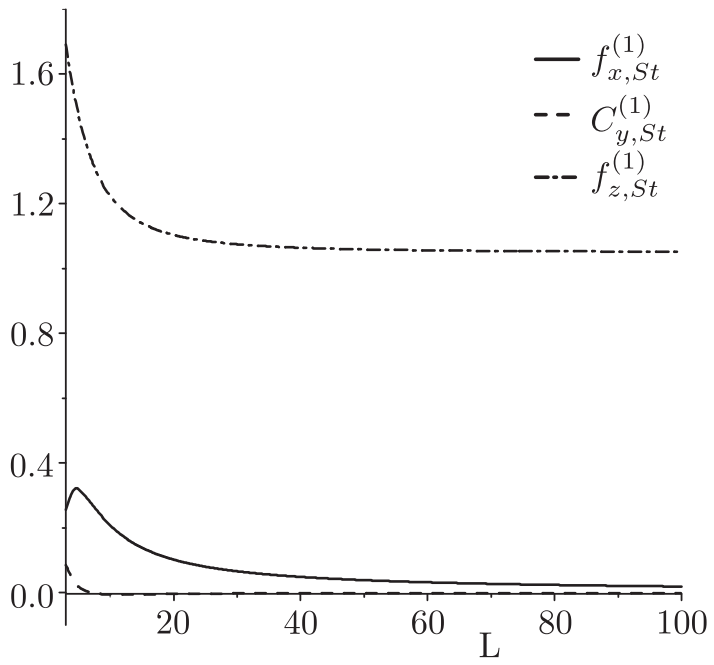

(a)

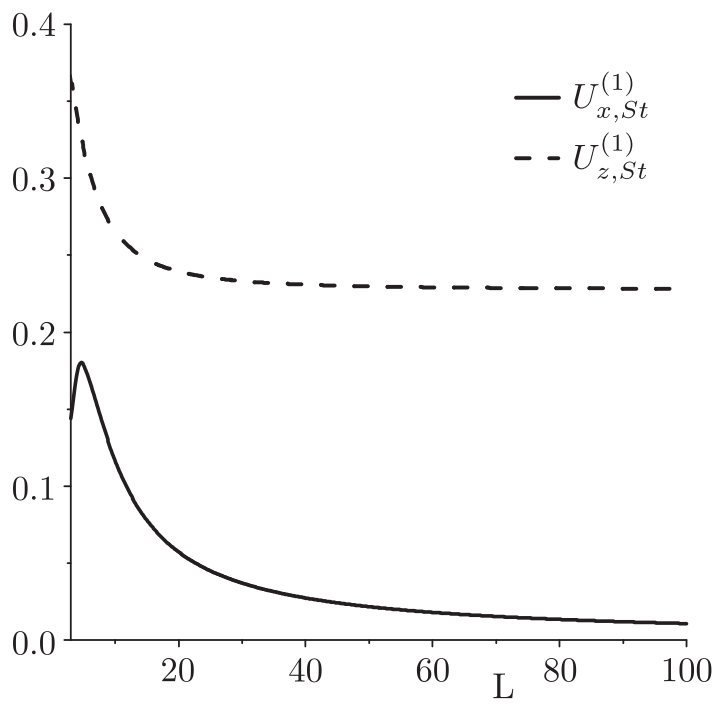

(b)

Fig. 3. (a) Lift, torque and drag coefficients and (b) Horizontal and vertical components of velocity; in terms of L. At $\mathrm{T}=0$ with $l / a=1.3$ and $\delta=3 / 4$.

\section{Conclusion}

This paper has studied the case of a spherical particle moving towards a corrugated wall in a fluid at rest. The flow around the sphere during its movement is described by Stokes flow with a low Reynolds number. By an asymptotic method, based on the hypothesis of small roughness amplitudes, coupled with the Lorentz reciprocity theorem, the analytical expressions of the force, torque and kinematic torsor due to roughness are determined without needing to compute the fields of velocity and pressure of second order. 


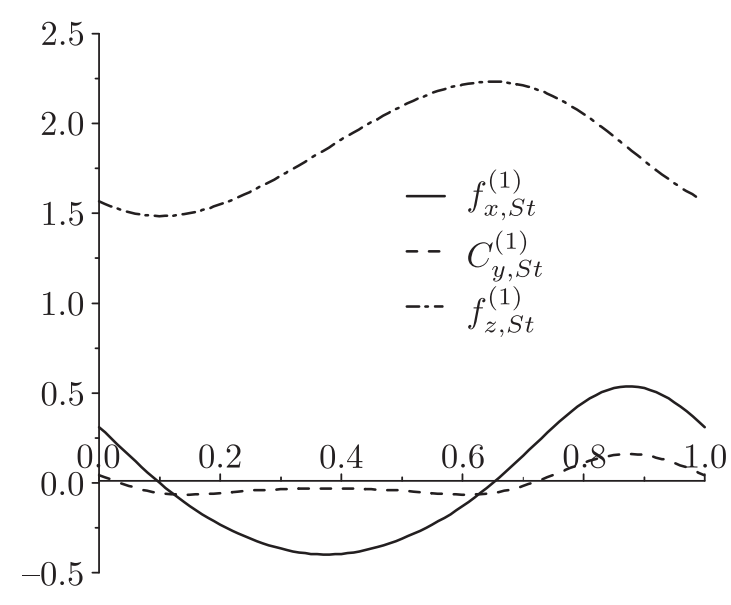

(a)

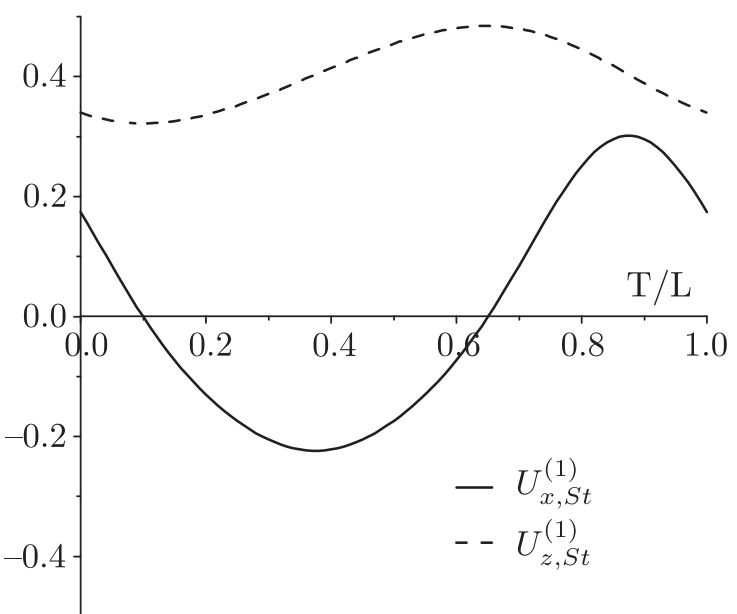

(b)

Fig. 4. (a) Lift, torque and drag coefficients and (b) Horizontal and vertical components of velocity; in terms of $\mathrm{T}$. At $\mathrm{L}=4$ with $l / a=1.3$ and $\delta=3 / 4$.

Numerical results show that these effects are influenced by roughness parameters (amplitude $\varepsilon$ and period L) as well as the relative position of the sphere. As expected, the influence of roughness is very important when the particle near the roughness, this influence fades rapidly with the growth of the distance separating the particle and the wall.

The authors thank Prof. Mohamed Chaoui for useful discussions.

\section{References}

[1] Williams, Ph. S., Koch, Th., and Giddings, J. C., Characterization of Near-Wall Hydrodynamic Lift Forces Using Sedimentation Field-Flow Fractionation, Chem. Eng. Commun., 1992, vol.111, no.1, pp. 121-147.

[2] Pasol, L., Martin, M., Ekiel-Jeżewska, M.L., Wajnryb, E., Bławzdziewicz, J., and Feuillebois, F., Motion of Sphere Parallel to Plane Walls in a Poiseuille Flow. Application to Field-Flow Fractionation and Hydrodynamic Chromatography, Chem. Eng. Sci., 2011, vol. 66, no. 18, pp. 4078-4089.

[3] Beebe, D. J., Mensing, G. A., and Walker, G. M., Physics and Applications of Microfluidics in Biology, Annu. Rev. Biomed. Eng., 2002, vol. 4, no. 1, pp. 261-286.

[4] Priezjev, N.V., Darhuber, A. A., and Troian, S. M., Slip Behavior in Liquid Films on Surfaces of Patterned Wettability: Comparison between Continuum and Molecular Dynamics Simulations, Phys. Rev. E, 2005, vol. 71, no. 4, 041608, 11 pp.

[5] Feuillebois, F., Some Theoretical Results for the Motion of Solid Spherical Particles in a Viscous Fluid, Multiphase Sci. Technol., 1989, vol. 4, nos.1-4, pp. 583-789.

[6] O'Neill, M. E., A Slow Motion of Viscous Liquid Caused by a Slowly Moving Solid Sphere: An Addendum, Mathematika, 1967, vol. 14, no. 2, pp.170-172.

[7] O’Neill, M.E., A Slow Motion of Viscous Liquid Caused by a Slowly Moving Solid Sphere, Mathematika, 1964, vol.11, no. 1, pp. 67-74.

[8] Goldman, A. J., Cox, R. G., and Brenner, H., Slow Viscous Motion of a Sphere Parallel to a Plane Wall: 1. Motion through a Quiescent Fluid, Chem. Eng. Sci., 1967, vol. 22, no. 4, pp.637-651.

[9] Chaoui, M. and Feuillebois, F., Creeping Flow around a Sphere in a Shear Flow Close to a Wall, Q. J. Mech. Appl. Math., 2003, vol. 56, no. 3, pp. 381-410. 
[10] Navier, C. L. M.H., Mémoire sur les lois du movement des fluids, Mém. Acad. Sci., 1827, vol.6, pp. 389-440.

[11] Maxwell, J. C., On Stresses in Rarified Gases Arising Inequalities of Temperature, Philos. Trans. Royal Soc., 1879, vol. 170, pp. 231-256.

[12] David, A. M. J., Kezirian, M. T., and Brenner, H., On the Stokes - Einstein Model of Surface Diffusion along Solid Surfaces: Slip Boundary Conditions, J. Colloid Interface Sci., 1994, vol.165, no.1, pp. 129-140.

[13] Elasmi, L., Singularity Method for Stokes with Slip Boundary Condition, J. Appl. Math., 2008, vol. 73, no. 5, pp. 724-739.

[14] Ghalya, N., Hydrodynamic Interactions between a Solid Particle and a Smooth Wall with Slip Condition of Navier, PhD Thesis, Palaiseau: École Polytechnique, 2012.

[15] Assoudi, R., Lamzoud, K., and Chaoui, M., Influence of the Wall Roughness on a Linear Shear Flow, FME Trans., 2019, vol. 46, no. 2, pp. 272-277.

[16] Falade, A. and Brenner, H., First-Order Wall Curvature Effects upon the Stokes Resistance of a Spherical Particle Moving in Close Proximity to a Solid Wall, J. Fluid Mech., 1988, vol. 193, pp. 533-568.

[17] Smart, J. R. and Leighton, D. T., Jr., Measurement of the Hydrodynamic Surface Roughness of Noncolloidal Spheres, Phys. Fluid, 1989, vol. 1, no. 1, pp. 52-60.

[18] Smart, J. R., Beimfohr, S., and Leighton, D. T., Jr., Measurement of the Translational and Rotational Velocities of a Noncolloidal Sphere Rolling Down a Smooth Inclined Plane at Low Reynolds Number, Phys. Fluid, 1993, vol. 5, no. 1, pp. 13-24.

[19] Lecoq, N., Anthore, R., Cichocki, B., Szymczak, P., and Feuillebois, F., Drag Force on a Sphere Moving Towards a Corrugated Wall, J. Fluid Mech., 2004, vol.513, pp. 247-264.

[20] Lecoq, N., Boundary Conditions for Creeping Flow along Periodic or Random Rough Surfaces, Experimental and Theoretical Results, J. Phys. Conf. Ser., 2012, vol. 392, no. 1, 012010, 19 pp.

[21] Assoudi, R., Chaoui, M., Feuillebois, F., and Allouche, H., Motion of a Spherical Particle along a Rough Wall in a Shear Flow, Z. Angew. Math. Phys., 2018, vol. 69, no. 5, Art. 112, 30 pp.

[22] Pasol, L., Chaoui, M., Yahiaoui, S., and Feuillebois, F., Analytical Solution for a Spherical Particle near a Wall in Axisymmetrical Polynomial Creeping Flows, Phys. Fluids, 2005, vol. 17, no. 7, 073602, 13 pp.

[23] Bernner, H., The Slow Motion of a Sphere through a Viscous Fluid towards a Plane Surface, Chem. Eng. Sci., 1961, vol. 16, nos. 3-4, pp. 242-251. 